\title{
Repeated vitrification/warming of human sperm gives better results than repeated slow programmable freezing
}

\author{
Teraporn Vutyavanich, Worashorn Lattiwongsakorn, Waraporn Piromlertamorn and Sudarat Samchimchom
}

In this study, we compared the effects of repeated freezing/thawing of human sperm by our in-house method of rapid freezing with slow programmable freezing. Sperm samples from 11 normozoospermic subjects were processed through density gradients and divided into three aliquots: non-frozen, rapid freezing and slow programmable freezing. Sperm in the rapid freezing group had better motility and viability than those in the slow freezing group $(P<0.01)$ after the first, second and third cycles of freezing/thawing, but there was no difference in morphology. In the second experiment, rapid freezing was repeated three times in 20 subjects. The samples from each thawing cycle were evaluated for DNA fragmentation using the alkaline comet assay. DNA fragmentation began to increase considerably after the second cycle of freezing/thawing, but to a level that was not clinically important. In the third experiment, rapid freezing was done repeatedly in 10 subjects, until no motile sperm were observed after thawing. The median number of repeated freezing/thawing that yielded no motile sperm was seven (range: 5-8, mean: 6.8). In conclusion, we demonstrated that repeated freezing/thawing of processed semen using our rapid freezing method gave better results than standard slow programmable freezing. This method can help maximize the usage of precious cryopreserved sperm samples in assisted reproduction technology.

Asian Journal of Andrology (2012) 14, 850-854; doi:10.1038/aja.2012.106; published online 15 October 2012

Keywords: comet assay; cryopreservation; DNA fragmentation; repeated freezing; sperm

\section{INTRODUCTION}

Intracytoplasmic sperm injection (ICSI) allows the use of a single motile sperm to inseminate an oocyte. When ICSI is performed, non-motile and even dead sperm can be used to inseminate an oocyte. ${ }^{1}$ It is now possible to cryopreserve severe oligozoospermic samples for subsequent use in ICSI treatment. ${ }^{2}$ ICSI also creates a considerable interest in refreezing and later re-use of sperm from those who have a limited number of cryopreserved sperm samples. ${ }^{3}$ Such a policy will benefit men who deposit sperm before vasectomy or cytotoxic treatment for cancer, and those with major medical illnesses or sexual dysfunction, as they may not be able to produce more samples. ${ }^{4}$ In a donor insemination program, refreezing increases the cost-effectiveness by reducing the expense to patients. More importantly, it reassures the availability of the same donor sperm for couples, who wish to have a second child. ${ }^{4}$

At least five studies ${ }^{3-7}$ have reported the effects of repeated freezing/ thawing on sperm motility, viability and DNA damage. Most of them used slow programmable freezing $(\mathrm{SPF})^{4,5}$ or liquid nitrogen vapor/ slush techniques. ${ }^{3,5-7}$ Recently, our team developed a new technique of rapid sperm freezing, which has several advantages over conventional slow freezing. The method does not require a programmable freezer. It is simple and consumes much less time, and the sperm survival and motility after cryopreservation are markedly improved. ${ }^{8}$

We hypothesized that our new method of sperm freezing would yield better survival and motility of sperm after repeated cycles of freezing/ thawing than conventional slow freezing. This study was, therefore, conducted to test our hypothesis. We also examined the effects of three cycles of freezing/thawing on DNA fragmentation and the number of possible repeated freezing/thawing cycles until no motile sperm were observed. The results of this study will help to maximize the efficient use of cryopreserved sperm samples for assisted conception treatment.

\section{MATERIALS AND METHODS}

Collection and preparation of semen samples prior to freezing The Ethics Committee of the Faculty of Medicine, Chiang Mai University approved the use of human sperm in this study (protocol No. 034/2553). Sperm samples were obtained from partners of infertile patients who gave written informed consent for the use of their sperm for research. These couples were undergoing infertility evaluation at Maharaj Nakorn Chiang Mai University Hospital in Chiang Mai, Thailand. The men produced sperm by masturbation after abstinence for 2-7 days. A routine semen analysis was performed within one hour of collection, according to the methods described by the World Health Organization. ${ }^{9}$ Sperm samples with parameters in the upper range of normality (concentration $>20$ million per $\mathrm{ml}$, and at least $50 \%$ progressive motility), were included in the study.

Sperm samples were layered on top of $80 \% / 40 \%$ discontinuous Percoll gradients (Pharmacia FineChemical AB, Uppsala, Sweden) and centrifuged at $300 \mathrm{~g}$ for ten minutes. The pellet was resuspended in Earle's balanced salts solution (EBSS) supplemented with $0.3 \%$ human serum albumin, $0.03 \mathrm{~mol} \mathrm{l}^{-1}$ sodium pyruvate and $0.01 \mathrm{~mol} \mathrm{l}^{-1} 4$-(2-hydroxyethyl)1-piperazineethanesulfonic acid, and centrifuged at $200 \mathrm{~g}$ for five minutes. After two washes, the final pellet was suspended in $400 \mu \mathrm{l}$ of EBSS. 


\section{Repeated slow programmable freezing and thawing}

An equal volume of warm $\left(37^{\circ} \mathrm{C}\right)$ sperm cryopreservation medium (SpermFreeze; FertiPro NV, Beernem, Belgium) was added dropwise to an aliquot of processed sperm sample. The mixture was loaded into a $0.25-\mathrm{ml}$ straw and left at room temperature for $10 \mathrm{~min}$. The straw was inserted into a programmable freezer (Planer Kryo 10 series III) and cooled at a rate of $-1{ }^{\circ} \mathrm{C}$ per min from $20{ }^{\circ} \mathrm{C}$ to $-5{ }^{\circ} \mathrm{C}$. The straw was then cooled at a rate of $-10^{\circ} \mathrm{C}$ per min to $-80^{\circ} \mathrm{C}$ per min. After a holding for $5 \mathrm{~min}$ at this temperature, the straw was plunged into liquid nitrogen.

After greater than or equal to one week of storage in liquid nitrogen, the samples were thawed by plunging the straw into water bath at $25-28{ }^{\circ} \mathrm{C}$. The thawed sample was first diluted with $0.5 \mathrm{ml}$ of EBSS supplemented with human serum albumin, pyruvate and 4-(2-hydroxyethyl)-1-piperazineethanesulfonic acid, and the remaining $3.5 \mathrm{ml}$ of supplemented EBSS was added gradually over a period of 3-4 min. The suspension was then centrifuged at $250 \mathrm{~g}$ for $5 \mathrm{~min}$ to remove the cryoprotectant. Post-thaw pellet was resuspended in $0.4 \mathrm{ml}$ of EBSS with supplements, and $0.1 \mathrm{ml}$ was removed and immediately assessed for sperm kinematics and viability. The remaining $0.3 \mathrm{ml}$ was cryopreserved by adding sperm cryopreservation medium a second time, as previously described.

After storage for 2-5 days, the sample was thawed and the cryoprotectant was removed. A small aliquot $(0.1 \mathrm{ml})$ from the second thaw was assessed for sperm kinematics and viability. The remaining sample $(0.3 \mathrm{ml})$ was then cryopreserved for a third time and thawed few days later. An aliquot from the third thaw was similarly studied for kinematics and viability.

\section{Repeated rapid freezing (RF)}

Our method of sperm freezing was previously described. ${ }^{8}$ In brief, the aliquot was mixed dropwise with an equal volume of cold cryopreservation medium $\left(4^{\circ} \mathrm{C}\right)$. The mixture was loaded into a pre-cooled 0.25 $\mathrm{ml}$ straw and left to incubate at $4{ }^{\circ} \mathrm{C}$ for $10 \mathrm{~min}$ in a refrigerator. The cryopreservation medium for rapid freezing was a modified human sperm preservation medium, ${ }^{10}$ in which $50 \mathrm{mmol} \mathrm{l}^{-1}$ of sucrose was replaced by $100 \mathrm{mmol} \mathrm{l}^{-1}$ of trehalose. The concentration of glycerol was decreased to $10 \%$, whereas that of human serum albumin was increased 20-fold. After $10 \mathrm{~min}$, the straw was inserted into a hole in a pre-cooled homemade aluminum block, previously immersed in liquid nitrogen for $20 \mathrm{~min}$ before use. The aluminum block remained immersed in liquid nitrogen during the whole process of freezing. The straw was held in the aluminum block for 3-5 min before transfer into a liquid nitrogen storage dewar.

Thawing was done in the same way as that described for the slow freezing technique. The thawed sample was washed with EBSS to remove the cryoprotectant. A small aliquot was removed for kinematic and survival study and the remaining sample were again re-frozen as previously described. After 3-7 days, the same procedure of thawing and freezing was done, until three cycles of freezing/thawing were completed.

\section{Determination of sperm motility parameters}

A 5- $\mu$ l volume of sample was loaded into a disposable counting chamber $(20-\mu \mathrm{m}$ depth; Leja, Niew-Vennep, The Netherlands) for the evaluation of sperm motility and kinematics of movement, using a computer-aided semen analyzer (CASA; HTM-IVOS, Hamilton Thorne Biosciences, Beverly, MA, USA), equipped with a clinical human motility program version 12 . The following parameter settings were used: frame rate, $60 \mathrm{~Hz} ; 30$ acquisition frames; straightness threshold, $80 \%$; minimum contrast, 80 ; minimum cell size, 3 pixels; non-motile head size, 6 pixels; non-motile head intensity, 160; illumination intensity, 2128; magnification, $\times 1.9$; temperature, $37{ }^{\circ} \mathrm{C}$; and chamber depth, $20 \mu \mathrm{m}$. At least 400 sperm trajectories were analyzed.

\section{Determination of sperm morphology}

A CASA (HTM-IVOS) was used to evaluate Diff-Quick stained slides for sperm morphology. Strict criteria were employed to analyze at least 200 sperm per sample, using Dimension Strict Morphology software on HTM-IVOS CASA. This program has been shown to give excellent repeatability of normal and abnormal cells, and to agree well with the manual strict criteria. ${ }^{11}$

\section{Determination of sperm viability}

Sperm viability was assessed by staining with $0.5 \%$ eosin-Y. ${ }^{9}$ After $1-2$ min, 100 sperm or more were counted as stained (dead) or unstained (viable).

\section{Determination of sperm DNA integrity by modified alkaline single-} cell gel electrophoresis (comet) assay

The status of sperm DNA was evaluated using a modified alkaline single-cell gel electrophoresis (comet) assay, as described by Hughes et al. ${ }^{12}$ and Donnelly et al. ${ }^{13}$ In brief, the method involves embedding the sperm in agarose, lysing the membranes and breaking down the protein matrices. Fragmented strands of DNA are drawn out by electrophoresis to form a comet 'tail', leaving a 'head' of intact DNA. The amount of intact and damaged DNA is quantified using an epifluorescence microscope, equipped with an excitation filter $(515-560 \mathrm{~nm})$, $100 \mathrm{~W}$ mercury lamp and barrier filter $(590 \mathrm{~nm})$. At least 100 sperm were captured and analyzed by image analysis software (LUCIA Comet Assay; Laboratory Imaging, Prague, Czeck Republic). Our intra-assay coefficient variation for this assay was $7 \%$.

\section{Experimental design (Figure 1)}

Experiment 1: Comparison of repeated freezing/thawing of sperm, using the SPF and RF techniques (11 subjects). In this experiment, we divided processed sperm samples into three aliquots. The first aliquot was not frozen, and was immediately assessed for sperm kinematics, morphology and viability. The second and third aliquots were cryopreserved by the SPF and our in-house method of RF, respectively. Three cycles of repeated freezing/thawing were performed. We assessed sperm viability, motility (World Health Organization grade $\mathrm{a}$ and $\mathrm{b}$ movement), morphology and kinematics immediately after each freeze/thaw cycles.

Experiment 2: Effect of three repeated freezing/thawing cycles by the RF technique on sperm DNA fragmentation. Sperm samples from 20 men were processed and divided into two aliquots. One aliquot was not frozen and was immediately assessed for sperm kinematics, viability and DNA fragmentation by the alkaline comet assay. Another aliquot was subjected to three cycles of rapid freezing/thawing. A $0.1 \mathrm{ml}$ aliquot from each thaw was assessed for sperm kinematics, viability, morphology and DNA fragmentation.

Experiment 3: Sperm samples from 10 men were processed and subjected to repeated cycles of RF until no motile sperm were observed.

\section{Statistical analysis}

We used STATA program version 8.2 (College Station, TX, USA) to perform all the statistical analyses. Data were expressed as mean \pm standard deviation (s.d.). A normality test was performed using the Shapiro-Wilk test. Mean percentages of motility, normal morphology, sperm kinematics, viability and DNA fragmentation before and after each freezing/thawing 


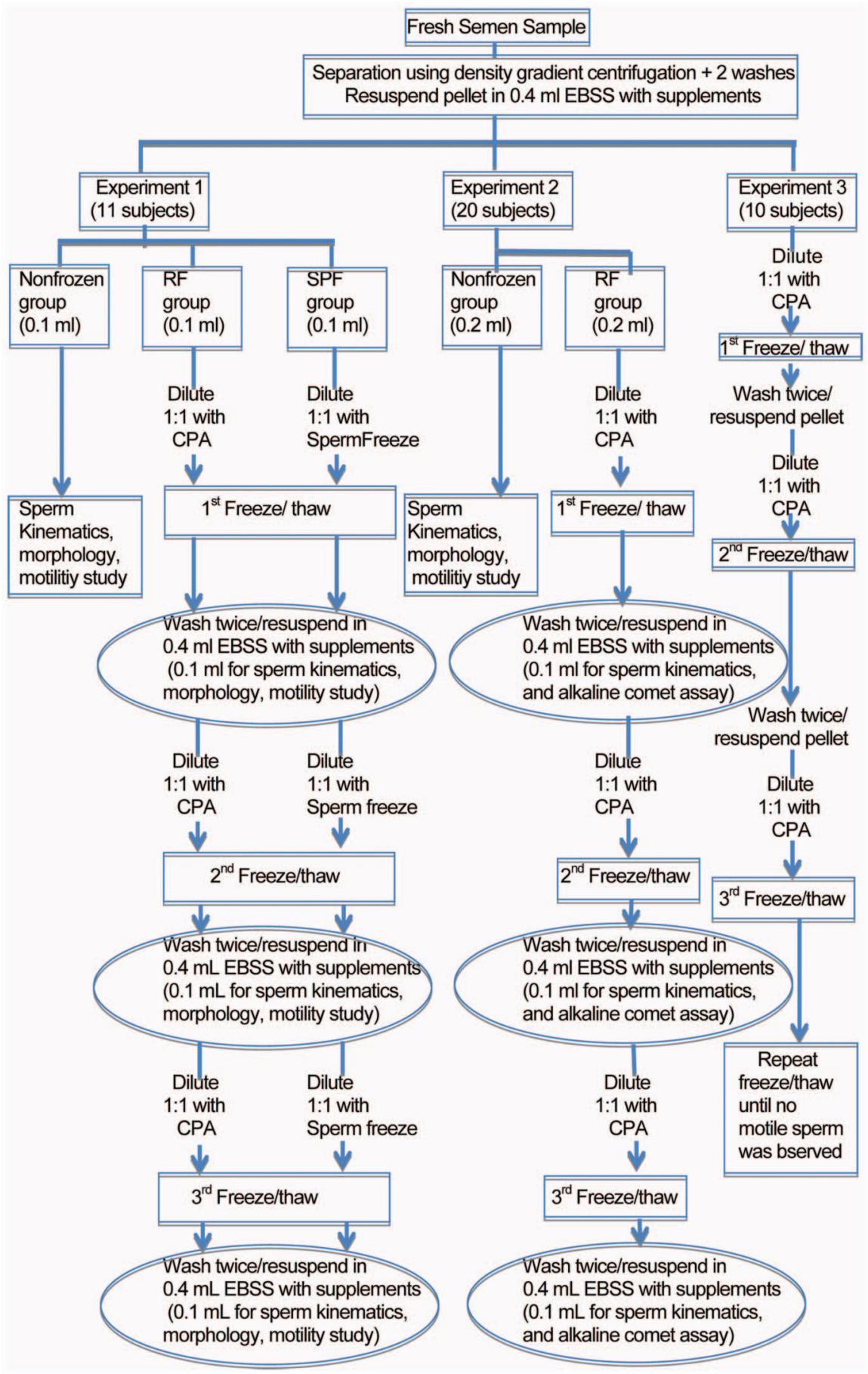

Figure 1 Diagram of methods. CPA, cryoprotective agent; EBSS, Earle's balanced salts solution; RF, repeated rapid freezing; SPF, slow programmable freezing.

cycle were compared by repeated measure ANOVA or Friedman nonparametric repeated-measure tests, as appropriate. A comparison was considered to be statistically significant if the $P$ value was $<0.05$.

\section{RESULTS}

\section{Experiment 1}

The mean age \pm s.d. of 11 subjects in this study was $31.2 \pm 5.6$ years. Sperm parameters were: volume, $2.1 \pm 0.9 \mathrm{ml}$; concentration, $75 \times 10^{6} \pm 22 \times 10^{6}$ per ml; motility, $72 \% \pm 11 \%$; viability, $82 \% \pm 8 \%$; and normal morphology, $22 \% \pm 9 \%$. The average path velocity (VAP), straight line velocity
(VSL), curvilinear velocity (VCL), amplitude of lateral head displacement (ALH) and linearity (LIN) are shown in Table 1. After each freezing/ thawing cycle, sperm viability and motility in the SPF group decreased more than those in the RF group, but there was no meaningful difference in sperm morphology and LIN between the two groups (Table 1).

\section{Experiment 2}

The mean age \pm s.d. of the 20 subjects in this study was $34.7 \pm 7.6$ years. Sperm parameters were: volume $2.9 \pm 0.8 \mathrm{ml}$; concentration, $68 \times 10^{6} \pm 24 \times 10^{6}$ per $\mathrm{ml}$; and motility $61 \% \pm 10 \%$. 
Sperm motility decreased by $18.5 \%, 53.3 \%$ and $69.1 \%$ after the first, second and third freeze/thaw cycles, respectively (Friedman test; $P<0.001)$. Sperm viability decreased by $20.4 \%, 38.9 \%$ and $54.6 \%$ after the first, second and third cycles, respectively (Friedman test; $P<0.001$ ). Cryopreservation caused a decrease (Friedman test; $P<0.01)$ in VAP, VSL and VCL, but it had little effect on ALH and LIN (Friedman test; $P>0.05$ ) (Table 2). Sperm DNA integrity was affected by repeated freezing/thawing as evidenced by considerable changes in the comet assay of sperm head and tail DNA, and tail moment (Table 2). Moreover, there was high variability in sperm DNA fragmentation between individual cases $(P<0.0001)$.

\section{Experiment 3}

Ten subjects in this study had a mean \pm s.d. age of $32.8 \pm 6.9$ years Sperm parameters were: volume, $2.6 \pm 0.5 \mathrm{ml}$; concentration, $69 \times 10^{6} \pm$ $23 \times 10^{6}$ per ml; motility, $84 \% \pm 4 \%$; and viability $87 \% \pm 3 \%$. The mean percentages \pm s.d. of sperm motility and viability after the first to the eighth cycle of freezing/thawing are shown in Table 3 . The median number of repeated freezing/thawing cycles that yielded no motile sperm was seven (range: 5-8; mode: 6, 7 and 8; mean: 6.8).

\section{DISCUSSION}

After three cycles of repeated freezing/thawing, the RF method gave better sperm survival and motility than the SPF. We washed the sperm and added fresh cryoprotectant in between each freeze-thaw cycle. This process could induce repeated cycles of osmotic shock to sperm,

Table 1 Repeated freezing/thawing cycles in the rapid (RF) and slow programmable freezing (SPF) groups $(n=11)$

\begin{tabular}{|c|c|c|c|}
\hline Freeze/thaw cycle no. & $R F$ & $S P F$ & $\mathrm{P}^{a}$ \\
\hline \multicolumn{4}{|l|}{ First cycle } \\
\hline Viability (\%) & $66.8 \pm 7.2$ & $55.4 \pm 8.6$ & 0.003 \\
\hline Morphology (\%) & $15.5 \pm 5.4$ & $19.8 \pm 9.9$ & 0.213 \\
\hline Motility (\%) & $66.3 \pm 12.4$ & $38.2 \pm 14.4$ & 0.002 \\
\hline VAP & $65.7 \pm 7.4$ & $52.2 \pm 8.9$ & 0.004 \\
\hline VSL & $55.7 \pm 7.8$ & $43.5 \pm 7.9$ & 0.006 \\
\hline VCL & $112.0 \pm 12.6$ & $95.6 \pm 1.3$ & 0.024 \\
\hline $\mathrm{ALH}$ & $5.4 \pm 0.9$ & $4.9 \pm 0.5$ & 0.151 \\
\hline LIN & $49.3 \pm 5.9$ & $46.6 \pm 6.1$ & 0.197 \\
\hline \multicolumn{4}{|l|}{ Second cycle } \\
\hline Viability (\%) & $54.1 \pm 5.2$ & $27.6 \pm 7.6$ & 0.000 \\
\hline Morphology (\%) & $16.8 \pm 4.9$ & $15.2 \pm 8.0$ & 0.572 \\
\hline Motility (\%) & $30.9 \pm 10.4$ & $14.0 \pm 5.9$ & 0.001 \\
\hline VAP & $51.0 \pm 7.7$ & $39.2 \pm 7.4$ & 0.000 \\
\hline VSL & $44.3 \pm 7.7$ & $32.2 \pm 6.3$ & 0.000 \\
\hline VCL & $94.1 \pm 11.9$ & $78.8 \pm 17.7$ & 0.005 \\
\hline $\mathrm{ALH}$ & $4.3 \pm 0.7$ & $4.5 \pm 1.5$ & 0.775 \\
\hline LIN & $47.2 \pm 6.8$ & $43.6 \pm 9.0$ & 0.193 \\
\hline \multicolumn{4}{|l|}{ Third cycle } \\
\hline Viability (\%) & $40.8 \pm 12.9$ & $14.2 \pm 4.6$ & 0.000 \\
\hline Morphology (\%) & $16.3 \pm 6.9$ & $11.8 \pm 6.2$ & 0.129 \\
\hline Motility (\%) & $18.4 \pm 8.7$ & $4.1 \pm 2.6$ & 0.000 \\
\hline VAP & $47.3 \pm 10.6$ & $20.2 \pm 13.7$ & 0.001 \\
\hline VSL & $39.4 \pm 7.9$ & $16.9 \pm 11.6$ & 0.001 \\
\hline VCL & $93.7 \pm 23.5$ & $42.2 \pm 29.2$ & 0.002 \\
\hline ALH & $4.9 \pm 1.8$ & $1.6 \pm 2.3$ & 0.004 \\
\hline LIN & $43.9 \pm 8.8$ & $39.3 \pm 25.9$ & 0.557 \\
\hline
\end{tabular}

Abbreviations: ALH, amplitude of lateral head displacement ( $\mu \mathrm{m})$; LIN, linearity (\%); VAP, average path velocity ( $\left.\mu \mathrm{m} \mathrm{s}^{-1}\right)$; VCL, curvilinear velocity $\left(\mu \mathrm{m} \mathrm{s}^{-1}\right)$; VSL, straight line velocity $\left(\mu \mathrm{m} \mathrm{s}^{-1}\right)$.

Values are expressed as means \pm s.d.

${ }^{a}$ Friedman non-parametric tests. in addition to the mechanical damage and oxidative stress from centrifugation. ${ }^{14,15}$ Previous studies, that employed similar washing step, ${ }^{4-6}$ consistently reported sperm motility as low as $2.0 \%-5.5 \%$ after three repeated cycles of freezing/thawing by slow programmable or conventional liquid nitrogen vapor freezing. This was in agreement with our study in the SPF group (motility: $4.1 \%$ ). In contrast, the study that refroze the samples in their original cryoprotectant without washing in between showed a better motility, which could be as high as $11.4 \%{ }^{7}$ Although not directly comparable, the result was still inferior to our repeated freezing/thawing by the RF method (motility: 18\%).

With CASA, an objective assessment of sperm kinematics can be performed to better determine which parameter may be more indicative of sublethal injury to human sperm. Previous studies concluded that the percentage of progressive motility, VAP, VSL and VCL but not $\mathrm{ALH}$ and LIN, were sensitive and useful indicators to determine adverse effects of toxic agents on human ${ }^{16}$ and rat sperm motion. ${ }^{17}$ By time-lapse photography, McLaughlin et al. ${ }^{18}$ also observed the average velocity and the percentage of progressive motility, but not $\mathrm{ALH}$, were indicative of cryodamage to human sperm. In our study (experiments 1 and 3), we found a similar adverse effect of cryoinjury on sperm kinematics, after three cycles of repeated freezing/thawing in the RF group. As LIN is defined as the ratio of VSL to VCL, the small changes observed in our study after repeated freezing/thawing suggested a decrease of both VSL and VCL to nearly the same extent.

In the second experiment, we focused our attention on the extent of DNA damage after three cycles of repeated freezing/thawing by the RF method. We used the alkaline comet assay as it allowed for sensitive detection of both single and double-stranded DNA breaks. ${ }^{19,20}$ In a recent study, ${ }^{21}$ the test had been shown to be useful for both the diagnosis of male factor infertility and prediction of in vitro fertilization outcome. The percentage of migrated DNA (7.2\% tail DNA) before freezing in our study was compatible with the background damage obtained by others under a similar condition $(\sim 10 \%$ tail DNA). ${ }^{20,22}$ It was reassuring that after three cycles of repeated freezing/thawing, the mean level of DNA fragmentation by the alkaline comet assay was still below the diagnostic threshold of $25 \%$, that predicted subfertility and below $42 \%$ that predicted failure to achieve a pregnancy after in vitro fertilization. ${ }^{21}$ In our study, a substantial

Table 2 Sperm parameters before and after each freezing/thawing cycle $(n=20)$

\begin{tabular}{|c|c|c|c|c|c|}
\hline \multirow[b]{2}{*}{ Parameters } & \multirow[b]{2}{*}{ Pre-freeze } & \multicolumn{3}{|c|}{ Cycle of freezing/thawing (F/T) } & \multirow[b]{2}{*}{$\mathrm{P}^{a}$} \\
\hline & & Cycle 1 & Cycle 2 & Cycle 3 & \\
\hline Viability (\%) & $85.6 \pm 9.2$ & $68.1 \pm 6.4$ & $52.3 \pm 9.9$ & $38.9 \pm 2.4$ & $<0.001$ \\
\hline Morphology (\%) & $20.6 \pm 6.1$ & $18.6 \pm 6.2$ & $18.0 \pm 4.4$ & $17.5 \pm 5.8$ & 0.018 \\
\hline Motility (\%) & $81.3 \pm 8.2$ & $66.3 \pm 9.4$ & $37.9 \pm 15.3$ & $25.1 \pm 7.2$ & $<0.001$ \\
\hline VAP & $69.2 \pm 13.4$ & $61.9 \pm 12.7$ & $48.3 \pm 13.1$ & $50.4 \pm 16.1$ & $<0.003$ \\
\hline VSL & $56.4 \pm 9.2$ & $52.0 \pm 11.8$ & $40.8 \pm 13.4$ & $43.1 \pm 15.9$ & $<0.003$ \\
\hline $\mathrm{VCL}$ & $120.2 \pm 34.9$ & $107.6 \pm 20.6$ & $87.7 \pm 17.9$ & $96.7 \pm 26.5$ & $<0.003$ \\
\hline ALH & $5.3 \pm 1.2$ & $5.0 \pm 0.8$ & $4.2 \pm 1.5$ & $4.5 \pm 1.9$ & 0.089 \\
\hline LIN & $49.1 \pm 7.9$ & $49.0 \pm 7.4$ & $46.1 \pm 9.6$ & $45.9 \pm 11.9$ & 0.764 \\
\hline \multicolumn{6}{|l|}{ Comet assay } \\
\hline Head DNA (\%) & $92.9 \pm 5.9$ & $91.0 \pm 6.5$ & $84.0 \pm 8.5$ & $75.5 \pm 12.8$ & $<0.001$ \\
\hline Tail DNA (\%) & $7.2 \pm 5.9$ & $9.0 \pm 6.5$ & $15.9 \pm 8.5$ & $24.5 \pm 12.8$ & $<0.001$ \\
\hline Tail moment & $13.6 \pm 16.0$ & $25.4 \pm 20.8$ & $32.6 \pm 18.9$ & $63.0 \pm 37.7$ & $<0.001$ \\
\hline
\end{tabular}

Abbreviations: ALH, amplitude of lateral head displacement ( $\mu \mathrm{m})$; LIN, linearity (\%); VAP, average path velocity ( $\left.\mu \mathrm{m} \mathrm{s}^{-1}\right) ; \mathrm{VCL}$, curvilinear velocity $\left(\mu \mathrm{ms}^{-1}\right) ; \mathrm{VSL}$, straight line velocity $\left(\mu \mathrm{m} \mathrm{s}^{-1}\right)$.

Values are expressed mean \pm s.d.

${ }^{a}$ Friedman nonparametric repeated measure tests. 
Table 3 Sperm motility and viability before and after each freezing/thawing cycle

\begin{tabular}{|c|c|c|c|c|c|c|c|c|c|c|c|c|c|c|c|c|c|c|}
\hline \multirow{3}{*}{$\frac{\text { Subject }}{\text { No. }}$} & \multicolumn{9}{|c|}{ Motility (\%) } & \multicolumn{9}{|c|}{ Viability (\%) } \\
\hline & \multicolumn{9}{|c|}{ Freezing/thawing cycle no. } & \multicolumn{9}{|c|}{ Freezing/thawing cycle no. } \\
\hline & 0 & 1 & 2 & 3 & 4 & 5 & 6 & 7 & 8 & 0 & 1 & 2 & 3 & 4 & 5 & 6 & 7 & 8 \\
\hline 2. & 80 & 55 & 50 & 34 & 16 & 5 & 0 & - & - & 82 & 63 & 53 & 36 & 21 & 17 & 3 & - & - \\
\hline 3. & 84 & 54 & 52 & 15 & 13 & 4 & 2 & 0 & - & 87 & 61 & 54 & 36 & 22 & 12 & 8 & 2 & - \\
\hline 4. & 92 & 55 & 41 & 20 & 10 & 0 & - & - & - & 92 & 55 & 41 & 20 & 10 & 0 & - & - & - \\
\hline 7. & 80 & 68 & 58 & 21 & 11 & 6 & 1 & 0 & - & 85 & 70 & 59 & 34 & 24 & 10 & 9 & 4 & - \\
\hline 8. & 85 & 70 & 35 & 16 & 10 & 3 & 1 & 0 & - & 90 & 72 & 36 & 28 & 22 & 18 & 10 & 3 & - \\
\hline 9. & 80 & 50 & 40 & 36 & 13 & 2 & 0 & - & - & 85 & 60 & 44 & 40 & 18 & 3 & 2 & - & - \\
\hline 10. & 81 & 70 & 40 & 24 & 14 & 8 & 4 & 2 & 0 & 85 & 72 & 47 & 32 & 20 & 12 & 9 & 5 & 3 \\
\hline Mean \pm s.d. & $83 \pm 4$ & $64 \pm 9$ & $46 \pm 8$ & $25 \pm 8$ & $14 \pm 3$ & $5 \pm 4$ & $2 \pm 3$ & $1 \pm 1$ & 0 & $87 \pm 3$ & $69 \pm 9$ & $51 \pm 9$ & $35 \pm 7$ & $22 \pm 6$ & $12 \pm 7$ & $8 \pm 4$ & $5 \pm 3$ & $5 \pm 2$ \\
\hline
\end{tabular}

increase in sperm DNA fragmentation only occurred after the second cycle of repeated freezing/thawing.

In the third experiment, three out of ten cases still had motile sperm after the seventh cycle of rapid freezing/thawing. A previous study of repeated freezing/thawing in liquid nitrogen vapor, without removing or adding fresh cryoprotectant, observed motile sperm in two out of seven normozoospermic specimens after the sixth cycle of freezing/ thawing, but none after the seventh cycle. ${ }^{7}$ Most other studies that employed conventional freezing with washing steps in between, however, hardly observed any motile sperm after only 3-5 cycles of repeated freezing/thawing. ${ }^{3-5}$

In conclusion, we demonstrated that repeated freezing/thawing of processed human sperm by our in-house method gave better results than standard slow programmable freezing. Our in-house method can help maximize the use of precious cryopreserved sperm samples in assisted reproduction technology. The method has been validated only on semen samples of high quality, which is normally the case for samples from sperm banking used for donor insemination. However, the method has not yet been proven valid for samples of patients with moderate or poor semen quality.

\section{AUTHOR CONTRIBUTIONS}

TV made substantial contributions to conception, design, analysis and interpretation of the data, and drafted the manuscript. WL participated in its design and coordination, carried out experiments 1 and 2, and helped to draft the manuscript. WP participated in the design, carried out experiments 2 and 3, and helped in data analysis and revising the manuscript critically for important intellectual content. SS participated in the acquisition of data in experiments $1-3$, and revised the manuscript critically for important intellectual content. All authors read and approved the final manuscript.

\section{COMPETING FINANCIAL INTERESTS}

All authors have no potential conflicts of interest, whether of a financial or other nature, with any pharmaceutical company.

\section{ACKNOWLEDGMENTS}

This research was supported by the Faculty of Medicine Endowment Fund for Medical Research, Chiang Mai University, Thailand (Grant No. 10/2551).
1 Tournaye H. Male factor infertility and ART. Asian J Androl 2012; 14: 103-8.

2 AbdelHafez F, Bedaiwy M, El-Nashar SA, Sabanegh E, Desai N. Techniques for cryopreservation of individual or small numbers of human spermatozoa: a systematic review. Hum Reprod Update 2009; 15: 153-64.

3 Polcz TE, Stronk J, Xiong C, Jones EE, Olive DL et al. Optimal utilization of cryopreserved human semen for assisted reproduction: recovery and maintenance of sperm motility and viability. J Assist Reprod Genet 1998; 15: 504-12.

4 Thomson LK, Fleming SD, Barone K, Zieschang JA, Clark AM. The effect of repeated freezing and thawing on human sperm DNA fragmentation. Fertil Steril 2010; 93: 1147-56.

5 Bandularatne $E$, Bongso $A$. Evaluation of human sperm function after repeated freezing and thawing. J Androl 2002; 23: 242-9.

6 Verza S Jr, Esteves SC. Feasibility of refreezing human spermatozoa through the technique of liquid nitrogen vapor. Int Braz J Urol 2004; 30: 487-93.

7 Verza S Jr, Feijo CM, Esteves SC. Resistance of human spermatozoa to cryoinjury in repeated cycles of thaw-refreezing. Int Braz J Urol 2009; 35: 581-90.

8 Vutyavanich T, Piromlertamorn W, Nunta S. Rapid freezing versus slow programmable freezing of human spermatozoa. Fertil Steril 2010; 93: 1921-8.

9 World Health Orgaization. WHO Laboratory Manual for the Examination and Processing of Human Semen. 5th ed. Geneva: WHO Press; 2010.

10 Mahadevan M, Trounson AO. Effect of cryoprotective media and dilution methods on the preservation of human spermatozoa. Andrologia 1983; 15: 355-66.

11 Kruger TF, du Toit TC, Franken DR, Menkveld R, Lombard CJ. Sperm morphology: assessing the agreement between the manual (strict criteria) and the sperm morphology analyzer IVOS. Fertil Steril 1995; 63: 134-41.

12 Hughes CM, Lewis SE, McKelvey-Martin VJ, Thompson W. A comparison of baseline and induced DNA damage in human spermatozoa from fertile and infertile men, using a modified comet assay. Mol Hum Reprod 1996; 2: 613-9.

13 Donnelly ET, O'Connell M, McClure N, Lewis SE. Differences in nuclear DNA fragmentation and mitochondrial integrity of semen and prepared human spermatozoa. Hum Reprod 2000; 15: 1552-61.

14 Mahfouz R, Sharma R, Lackner J, Aziz N, Agarwal A. Evaluation of chemiluminescence and flow cytometry as tools in assessing production of hydrogen peroxide and superoxide anion in human spermatozoa. Fertil Steril 2009; 92: 819-27.

15 John Morris G, Acton E, Murray BJ, Fonsea F. Freezing injury: the special case of the sperm cell. Cryobiology 2012; 64: 71-80.

16 Grizard G, Ouchchane L, Roddier H, Artonne C, Sion B et al. In vitro alachlor effects on reactive oxygen species generation, motility patterns and apoptosis markers in human spermatozoa. Reprod Toxicol 2007; 23: 55-62.

17 Kawaguchi T, Kawachi M, Morikawa M, Kazuta H, Shibata K et al. Key parameters of sperm motion in relation to male fertility in rats given alpha-chlorohydrin or nitrobenzene. J Toxicol Sci 2004; 29: 217-31.

18 McLaughlin EA, Ford WC, Hull MG. Motility characteristics and membrane integrity of cryopreserved human spermatozoa. J Reprod Fertil 1992; 95: 527-34.

19 Bajpayee M, Pandey AK, Parmar D, Dhawan A. Current status of short-term tests for evaluation of genotoxicity, mutagenicity, and carcinogenicity of environmental chemicals and NCEs. Toxicol Mech Methods 2005; 15: 155-80.

20 Speit GN, Vasquez M, Hartmann A. The comet assay as an indicator test for germ cell genotoxicity. Mutat Res 2009; 681: 3-12.

21 Simon L, Lewis SE. Sperm DNA damage or progressive motility: which one is the better predictor of fertilization in vitro? Syst Biol Reprod Med 2011; 57: 133-8.

22 Donnelly ET, McClure N, Lewis SE. The effect of ascorbate and $\alpha$-tocopherol supplementation in vitro on DNA integrity and hydrogen peroxide-induced DNA damage in human spermatozoa. Mutagenesis 1999; 14: 505-11. 